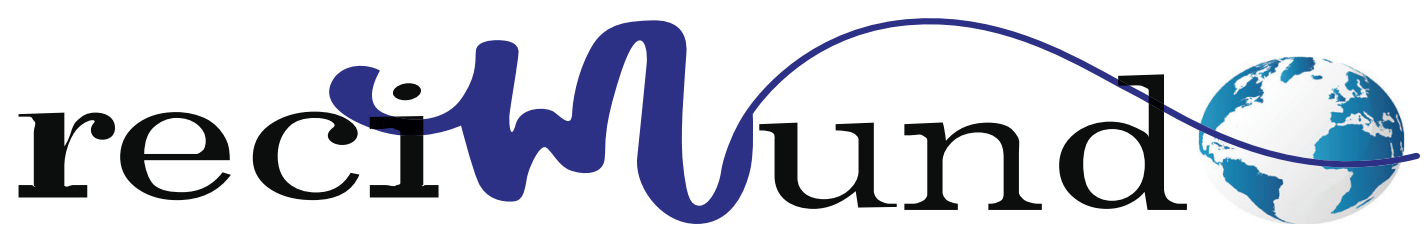

Revista Científica Mundo de la Investigación y el Conocimiento

DOI: 10.26820/recimundo/4.(4).octubre.2020.509-516

URL: http://recimundo.com/index.php/es/article/view/975

EDITORIAL: Saberes del Conocimiento

REVISTA: RECIMUNDO

ISSN: 2588-073X

TIPO DE INVESTIGACióN: Artículo de Revisión

CÓDIGO UNESCO: 32 Ciencias Médicas

PAGINAS: $509-516$

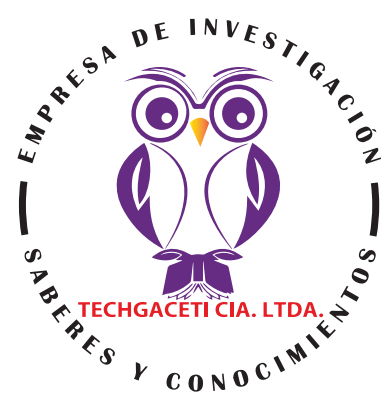

\title{
La diabetes y las complicaciones con el COVID-19
}

Diabetes and complications with COVID-19

Diabetes e complicações com o IDOC-19

Patricia Judith Pinos Robalino1; Patricia Segovia Palma²; María Jacqueline Cedeño Delgado3;

Efigenia Monserrate Gonzabay Bravo ${ }^{4}$

RECIBIDO: 10/11/2020 ACEPTADO: 26/11/2020 PUBLICADO: 13/12/2020

1. Doctora en Odontología; Diploma Superior en Diseño Curricular por Competencias; Magister en Diseño Curricular; Universidad de Guayaquil, Guayaquil, Ecuador; patricia.pinosr@ug.edu.ec; (ID https://orcid.org/0000-0001-7170-9381

2. Doctora en Odontología; Magister en Diseño Curricular; Diplomado en Docencia Superior; Universidad de Guayaquil, Guayaquil, Ecuador; patricia.segoviap@ug.edu.ec; iD https://orcid.org/0000-0003-2551-8036

3. Doctora en Odontología; Magister en Docencia Universitaria e Investigación Educativa; Diploma Superior en Diseño Curricular por Competencias; Universidad de Guayaquil, Guayaquil, Ecuador; maria.cedeñod@ug.edu.ec; (DD https://orcid. org/0000-0002-9865-0938

4. Doctora en Odontología; Magister en Docencia Universitaria e Investigación Educativa; Diploma Superior en Preparación de Multirradiculares; Universidad de Guayaquil, Guayaquil, Ecuador; efigenia.gonzabayb@ug.edu.ec; (DD https://orcid. org/0000-0001-5753-7747

CORRESPONDENCIA

Patricia Judith Pinos Robalino

patricia.pinosr@ug.edu.ec

Guayaquil, Ecuador

๑ RECIMUNDO; Editorial Saberes del Conocimiento, 2020 


\section{RESUMEN}

Los estudios epidemiológicos constataron la vulnerabilidad de los pacientes con Diabetes frente al COVID-19, y su alta probabilidad de generar complicaciones graves si no se logra un control glucémico óptimo según el caso. Muchos de estos pacientes al ingresar al hospital presentan frecuentemente cetoacidosis diabética severa lo cual podría complicarse críticamente, si no se actúa de manera oportuna. Muchas recomendaciones han sido dadas por expertos para manejar y/o prevenir esos casos que podrían formar parte de las estadísticas de mortalidad de este nuevo Coronavirus.

Palabras clave: Diabetes, COVID-19, Complicación, Riesgo, Coronavirus.

\section{ABSTRACT}

Epidemiological studies confirmed the vulnerability of Diabetes patients to COVID-19, and their high probability of generating serious complications if optimal glycemic control is not achieved depending on the case. Many of these patients upon admission to the hospital frequently present with severe diabetic ketoacidosis, which could be critically complicated if it is not acted upon in a timely manner. Many recommendations have been given by experts to manage and / or prevent those cases that could be part of the mortality statistics of this new Coronavirus.

Keywords: Diabetes, COVID-19, Complication, Risk, Coronavirus.

\section{RESUMO}

Estudos epidemiológicos confirmaram a vulnerabilidade dos doentes de Diabetes à COVID-19, e a sua elevada probabilidade de gerar complicações graves se não for alcançado um controlo glicémico óptimo, dependendo do caso. Muitos destes pacientes aquando da sua admissão no hospital apresentam frequentemente cetoacidose diabética grave, que poderia ser criticamente complicada se não fosse agida em tempo útil. Muitas recomendações foram dadas por especialistas para gerir e/ou prevenir os casos que poderiam fazer parte das estatísticas de mortalidade deste novo Coronavírus.

Palavras-chave: Diabetes, COVID-19, Complicação, Risco, Coronavírus. 


\section{Introducción}

La ansiedad y el estrés que ha producido la pandemia del COVID-19 en el mundo, constituyen de por sí un factor de riesgo, que logra bajar las defensas del organismo, peor aún lo será, para aquellos organismos que ya tienen que lidiar con patologías como la Diabetes, enfermedades cardiovasculares, o asma. Estas enfermedades crónicas han sido vigiladas en todos los estudios epidemiológicos desde el 11 de Marzo de 2020, cuando la OMS declaró la Pandemia por COVID-19, hasta la fecha. Se ha observado que estos casos que han presentado comorbilidades, resultan tener una alta tasa de mortalidad.

Probabilísticamente hablando, el riesgo de contraer la infección del Coronavirus, es el mismo para todo el mundo, porque depende de las condiciones a las que se exponga la persona. Sin embargo, si una persona que ya de por sí tiene su salud afectada por alguna enfermedad subyacente como la diabetes, se expone al virus, la posibilidad de contraerlo es elevada por su condición inmunológica debilitada, además su organismo tendría que ejercer mayor esfuerzo para recuperarse.

Las estadísticas han demostrado que la mayoría de las personas contagiadas con el COVID-19 se recuperan con facilidad, no obstante, esto no ocurre con los pacientes que padecen de enfermedades crónicas y contraen la infección por Coronavirus. Por lo general, estas personas pueden desarrollar complicaciones graves cuando se combina esta infección con su enfermedad subyacente, así lo confirman varias estadísticas epidemiológicas reportadas por todo el mundo:

- Las intensas investigaciones en China publicadas, después de los casos producidos en Wuhan, dieron a conocer que la tasa de mortalidad del COVID-19 era de $2 \%$, pero para personas que pa- decían enfermedades cardiovasculares era de $10 \%$ y de un $7 \%$ en diabéticos. (1)

- Las estadísticas preliminares del mes de marzo, reportadas por los Centros para el Control y la Prevención de Enfermedades, mostraron que del $89.3 \%$ de los casos de Coronavirus que requirieron hospitalización y atención especializada, padecían de una enfermedad subyacente, de los cuales el $28,3 \%$ eran pacientes diabéticos. (2)

- Según estudios realizados por la autoridad Nacional de Italia, publicados a finales de marzos, mostraron que el 99\% de muertes por COVID-19, fueron personas que ya padecían una enfermedad previa. "Más del 75 por ciento tenía presión arterial alta, alrededor de un 35 por ciento sufría diabetes y una tercera parte padecía enfermedades cardíacas." (3)

El presente artículo hace referencia a las complicaciones que podrían presentarse en aquellos pacientes diabéticos contagiados por esta nueva cepa de Coronavirus denominada COVID-19, y plantea el control de glucemia en estos pacientes contagiados para evitar dichas complicaciones de la enfermedad. Además, expone la importancia de que los profesionales de la salud exhorten a sus pacientes diabéticos (tipo 1 o tipo 2) a mantener el control estrictode su glucemia, explicándoles de forma clara la información que ellos necesitan saber para evitar males mayores.

\section{Metodología}

Desde una perspectiva cualitativa se realizó una exhaustiva revisión documental de la que se extrajo la información idónea, valedera y oportuna para el desarrollo referencial del material bibliográfico. Esto, dada la extensa información que se tiene en la actualidad sobre el COVID-19 y al mismo tiempo, un escueto contenido científico que valide de manera fáctica el efecto de este virus sobre las personas que padecen de

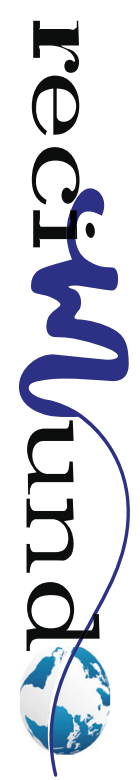


Diabetes o cualquier otra enfermedad subyacente, ya que todavía los hallazgos científicos sobre el mismo, parecen no estar muy claros aún.

Es por ello, que se ha revisado diferentes fuentes estadísticas epidemiológicas del COVID-19, tomando como valores de referencias las cifras dadas por la OMS (Organización Mundial de la Salud), para poder dar una demostración clínica sobre las complicaciones de los pacientes con Diabetes derivadas de ésta pandemia que ha aterrorizado al mundo entero.

Ciertamente, es obvio que cualquier persona con una patología crónica expuesta a cualquier enfermedad infecciosa es vulnerable, y el desarrollo de dicha enfermedad puede llegar a complicarse hasta la gravedad. Sin embargo, es necesaria una argumentación científica que explique y defina la fisiopatología detrás de todo este evento. En tal sentido, el enfoque documental usado en el presente artículo, va orientado a la descripción de las complicaciones del COVID-19 en pacientes que padecen cualquier tipo de Diabetes, basándose en la información y recomendaciones de un panel constituido por 19 expertos dirigidos por el Dr. Stefan R. Bornstein, del Centro Helmholtz en Múnich, y la Universidad Técnica de Desdén, en Alemania; también formaron parte de éste panel expertos de Europa, Estados Unidos, Asia, Australia y Sudamérica. El 23 de abril se publicaron dichas recomendaciones en una versión electrónica en TheLancet Diabetes \&Endocrinology (4).

\section{Resultados}

\section{Diabetes}

Para el 2014, la diabetes era una enfermedad crónica que afectaba a más de 442 millones de personas adultas en el mundo, esto es un 8,5 \% de la población mundial (5). Se diagnóstica cuando los valores de glucemia se presentan elevados (>125 mg/ dl o $>7 \mathrm{mmol} / \mathrm{l}$ ) en varias pruebas individuales, acompañado del siguiente cuadro clínico:

- Polidipsia

- Poliuria

- Polifagia

- Pérdida de peso inexplicable

- Presencia de cetonas en la orina (subproducto de la degradación muscular y de grasas que se produce por deficiencia o ausencia de insulina)

- Fatiga

- Irritabilidad

- Visión borrosa

- Llagas de cicatrización lenta

- Infecciones frecuentes (infecciones en las encías, en la piel, en la vagina). (6)

Referirse a la Diabetes tanto al término mismo como a la enfermedad al que hace referencia, implica, por un lado, un inexacto y polémico significado etimológico y lexicográfico desde su origen (7), y por el otro, una discusión parcialmente consensuada entre la comunidad científica, ya que la clasificación de la misma aún no es muy precisa (8)

Sin embargo, la mayoría de los centros de salud a nivel mundial, acatan la terminología propuesta por la OMS (9), donde se definen dos tipos, los cuales se referencian con números arábicos y no romanos para evitar confusiones:

Diabetes Tipo 1: Definida por la enfermedad autoinmune en la que el paciente no puede producir insulina para regular la glucemia porque su propio organismo ataca las células- $\beta$ delpáncreas, por lo que requiere de inyecciones de insulina para sobrevivir. Siempre se ha manifestado en la infancia, o en la juventud.

Diabetes tipo 2: Se produce cuando el organismo no produce suficiente insulina para regular la glucemia, la etiología generalmente está relacionada con malos hábitos 
alimenticios y sedentarismo, y otros factores de riesgos como obesidad e hipertensión, lo cual lleva a la degeneración de las células- $\beta$ pancreáticas. Anteriormente estaba asociada sólo a las personas adultas o de avanzada edad, pero en la actualidad los casos en la infancia comienzan a elevarse de manera preocupante. Este tipo de diabetes es controlable con farmacología oral, con la alimentación y ejercicio físico adecuados.

También se clasifica la Diabetes Gestacional, la cual se produce en algunos embarazos, y generalmente desaparece cuando termina el mismo. Sin embargo, es necesario que la mujer se chequee regularmente, para descartar la posibilidad de desarrollar la enfermedad.

En países de pocos recursos, es difícil diagnosticar y clasificar el tipo de diabetes $(\mathrm{Ha}$ excepción de la Diabetes Gestacional), por lo costosas y sofisticadas que son las pruebas de laboratorio, tal vez por ello la mortalidad prematura por diabetes (sin distinción entre sus tipos), es más elevada en estos países. (5)

La Diabetes Tipo 1 no puede prevenirse con la ciencia actual, pero la Diabetes Tipo 2 no sólo puede ser revertida en ciertos casos, sino también prevenida con un adecuado sistema de salud que concientice a las personas sobre la importancia de una saludable alimentación y la práctica de deportes o ejercicios como parte de su cotidianidad.

La Diabetes sin control, por si sola puede complicarse con el tiempo deteriorando el organismo, dañando nervios y tejidos, desarrollando Nefropatía, y Retinopatía. Actualmente, ante la pandemia del COVID-19, una persona con una Diabetes no controlada tiene baja probabilidad de recuperarse fácilmente de la infección si llega a contagiarse, y si además esta persona es de avanzada edad, el pronóstico tiende a ser negativo según los estudiosepidemiológicos realizados durante la epidemia en China (10).
Clínicamente no se puede establecer una explicación de cómo la Diabetes supone un factor de riesgos para contraer el COVID-19 ante su exposición, y de cómo llega a ser tan delicado el curso de la enfermedad en estos casos. Sin embargo, muchos estudios subclínicos han determinado cómo funciona el mecanismo por el cual los casos de pacientes Diabéticos son tan comunes también en otras infecciones por coronavirus, como el síndrome respiratorio agudo severo (SRAS) y el síndrome respiratorio del Medio Oriente (MERS-CoV) (11).

\section{Complicaciones de la diabetes con CO- VID-19}

Diferentes estudios epidemiológicos muestran como la incidencia de casos de COVID-19 en personas diabéticas, hipertensas, enfermedades respiratorias crónicas, enfermedades cardiovasculares y el cáncer, son factores de riesgo asociados a la mortalidad por Coronavirus.

Generalmente, todos los tipos de Diabetes tienen un mayor riesgo de infección debido a que el organismo diabético va perdiendo la capacidad de realizar fagocitosis, la quimiotaxis de neutrófilos y la inmunidad celular. No obstante, se ha observado en las estadísticas, mayor incidencia de casos de pacientes con Diabetes tipo 2, pero puede estar reflejando la prevalencia de los casos de personas mayores que ha caracterizado a esta pandemia.

En efecto, las complicaciones graves con mayor incidencia que presentan los pacientes diabéticos cuando se infectan por el Coronavirus son:

- Shock Séptico y la sepsis :En el 2016 la EuropeanSociety of IntensiveCare Medicine y la Society of CriticalCare Medicine definieron la Sépsiscomo "una disfunción orgánica que amenaza la vida debido a una respuesta desregulada del anfitrión ante la infección". (12) Esta

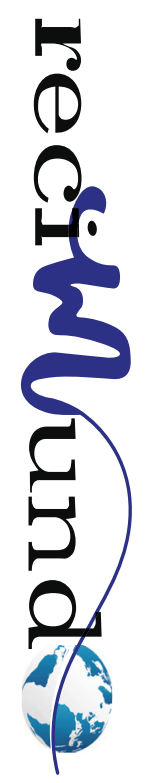


complicación gravela han sufrido algunos pacientes de coronavirus a causa de la cetoacidosis diabética, la cual estropea la ingesta de fluidos y los niveles de electrolitos, lo que es crucial para enfrentar la sepsis. El fallo multiorgánico es más probable en pacientes diabéticos y por lo general puede llegarse a un shock séptico, o la muerte.

- Síndrome de Distrés Respiratorio del adulto (SDRA): Según la conferencia europeo-americana de consenso de 1994, se definecomo "aquella situación o situaciones que alteran el intercambio de gases, con una relación entre la presión parcial de oxígeno arterial y la fracción inspirada de oxígeno ( $\mathrm{PaO} 2 / \mathrm{FiO} 2$ ) menor de 200, sin importar cuál sea el nivel de presión positiva al final de la espiración (PEEP), muestra infiltrados bilaterales en ambos campos pulmonares en la radiografía de tórax y una presión de enclavamiento pulmonar (PCP) menor de $18 \mathrm{mmHg}$ " (13). Los pacientes con Diabetes que se complican a este nivel, necesitando de oxigenoterapia y ventilación mecánica, necesitan un cuidado intensivo, que le garanticen el monitoreo estrictos de todas las constantes vitales, y demás valores metabólicos.

Este tipo de complicación requiere de atención especializada (UCl), y pocas veces se logra revertir la gravedad con la consecuente mortalidad. Sin embargo, gracias a los diferentes estudios epidemiológicos y subclínicos, se ha podido tener una primera aproximación que explica por qué se complica el paciente diabético infectado con el COVID-19, aunado a esto, se puede tener herramientas para evitar llegar a estas complicaciones.

Estos estudios plantean que existen por lo menos dos mecanismos que podrían explicar el proceso de infección del COVID-19 en organismo que padece de Diabetes:
1. El virus SARS-CoV-2 secuestra una vía endocrina que juega un papel importante en la regulación de la presión arterial, el metabolismo y la inflamación, con el objetivo de ingresar a sus células diana: La enzima convertidora de angiotensina 2 (ACE2). (14) Es decir, la ACE2 ha sido identificada como el receptor de la proteína espiga del coronavirus. La infección por COVID-19 reduce la acción de ACE2 induciendo daño celular, hiperinflamación e insuficiencia respiratoria.

Por otro lado, se demostró que la hiperglucemia crónica regula negativamente la expresión de ACE2 haciendo que las células sean vulnerables al efecto inflamatorio y dañino del virus. Además, la expresión de ACE2 en las células $\beta$ pancreáticas puede conducir a un efecto directo sobre la función de las células $\beta$, causando una reducción de insulina (15).

2. La relación entre el COVID-19 y la diabetes la establece la enzima dipeptidil peptidasa-4 (DPP-4), que ya se había identificado como receptor funcional cuando apareció el coronavirus humano Erasmus Medical Center (hCoV-EMC), el MERS.La DPP-4 aumenta la inflamación en la Diabetes tipo 2, lo que podría explicar por qué hay más incidencia de éste tipo de Diabéticos contagiados con COVID-19.

Aunque todavía las explicaciones de los mecanismos no son contundentes, han permitido desarrollar un manejo oportuno en los casos de pacientes diabéticos infectados por COVID-19. Y el punto más importante hacia donde convergen todas las teorías y estudios es al control de la Hiperglucemia.

En el presente artículo, se resume propuestas y recomendaciones dadas por un panel de expertos con las que coinciden consensualmente, hasta la fecha, con otros expertos médicos dedicados a dar soluciones a ésta crisis pandémica. El objetivo al alcanzar es un óptimo control de glicemia según el caso: 
Prevención Ambulatoria: Aunque debido a la cuarentena se ha producido un distanciamiento personal, se deben realizar campañas en todos los medios de comunicación y redes sociales para concientizar a esa población diabética y a sus familiares, para que realicen con responsabilidad el control de glucemia. Se debe mantener una glucemia entre 4 - 8 mmol/l (72 - $144 \mathrm{mg} / \mathrm{dl}$ ).

Pacientes Hospitalizados con Covid: Se sugiere un estricto control de Glucemia. Suspender los fármacos como la Metformina, para disminuir el riesgo de aumento de acidosis láctica; y también los inhibidores del cotransportador de sodio-glucosa, para disminuir el riesgo de acidosis metabólica. Todo ello, para el los casos de Diabetes tipo 2. De esta manera se reducen los riesgos de descompensación metabólica Núnca se debe disminuir la dosis de insulina, mientras no esté dado de alta. Se debe alcanzar una glucemia 4 - 10 mmol/l (72 - 180 mg/dl). Pacientes en UCI: Se debe Monitorear glucosa plasmática, electrólitos, $\mathrm{pH}$, cetonas en sangre o b-hidroxibutirato.Se debe prescribir insulinoterapia intravenosa temprana para evitar complicaciones como el síndrome de dificultad respiratoria aguda, hiperinflamación. Se debe lograr una glucemia de 4 - $10 \mathrm{mmol} / \mathrm{l}$ (72 - $180 \mathrm{mg} / \mathrm{dl})$, con posible ajuste ascendente del valor bajo, hasta alcanzar una cifra de $5 \mathrm{mmol} / \mathrm{l}(90 \mathrm{mg} / \mathrm{dl})$.

En general,

- Se debe mantener una Hemoglobina glucosilada< $53 \mathrm{mmol} / \mathrm{mol}(7 \%)$,

- Una monitorización continua de la glucosa/monitorización intermitente de la glucosa: tiempo en rango (3,9 - 10 $\mathrm{mmol} / \mathrm{l}$ ) > 70\% del tiempo (o > 50\% en pacientes críticos y de edad avanzada).

- Hipoglucemia < 3,9 mmol/l (<70 mg/dl): $<4 \%$ (< $1 \%$ en personas débiles y de edad avanzada).

- Se deben ajustar la dosis de insulina según las necesidades, las cuales pueden ser muy elevadas en personas con
COVID-19. Puede requerirse la infusión intravenosa de insulina.

- Se debe suspender el uso de inhibidores de la enzima convertidora de angiotensina, y antagonistas de receptor de angiotensina II.

- Se debe mantener el uso de Estatina que beneficierá a largo plazo y para inclinar la balanza hacia la tormenta de citocinas por los incrementos de rebote en la interleucina-6 y la interleucina-1b.

\section{Conclusiones}

Las investigaciones realizadas confirman que:

- Existe una mayor incidencia de pacientes con Diabetes que desarrollan complicaciones al contagiarse con el COVID-19.

- Existe una mayor su probabilidad para el ingreso a UCl de pacientes con Diabetes infectados por COVID-19. Estas complicaciones vienen sumadas a la descompensación metabólica causada por la hiperglucemia no controlada por dichos pacientes, haciendo necesario el ingreso a Cuidados intensivos ya que una cetoacidosis, puede derivar en una Sepsis o en un Síndrome de Distrés Respiratorio del adulto (SDRA).

Se ha demostrado que existen al menos dos vías de acceso y anclaje del Coronavirus en las células, una a través de la enzima convertidora de angiotensina 2 (ACE2), y la otra a través de la enzimadipeptidil peptidasa-4 (DPP-4). Aunque los estudios no se han aplicado en humanos, se cree que estas podrían ser la clave para bloquear el desarrollo del COVID-19 en el organismo.

Expertos de todo el mundo publicaron recomendaciones y ciertas directivas, con la finalidad de orientar a los representantes de la salud para que realicen su esfuerzo en prevenir y evitar complicaciones en estos casos vulnerables que ataca el Coonavirus.

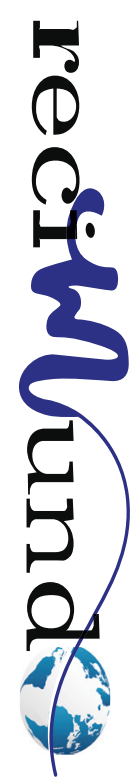


Todas las recomendaciones apuntan al control glucémico como única vía para no dejar descompensar al paciente Diabético. Para ello, es necesaria la administración de insulina como terapia importante, y suspender (en el caso de los pacientes con Diabetes Tipo 2) algunos medicamentos para disminuir el riesgo de acidosis láctica y cetoacidosis.

\section{Bibliografía}

1. Zhu L, She ZG, Cheng X, Guo J, Zhang BH, Li H. Association of Blood Glucose Control and Outcomes in Patients with COVID-19 and Pre-existing Type 2 Diabetes. Cell Metabolism. 2020 Apr; 21(3).

2. Center for Disease Control and Prevention. COVID-NET. [Online].; 2020 [cited 202058 [Accessed on DATE]. Available from: https://www.cdc. gov/mmwr/volumes/69/wr/mm6915e3.htm\#T1_ down.

3. Ebhardt T, Remondini C, Bertacche M. El 99\% de los fallecidos por coronavirus en Italia tenía otras enfermedades previas: estudio. 2020 Mar 20. Grupo Multimedia Lauman, SAPI de CV.

4. Bornstein S, Rubino, Khunti K, Mingrone G, Hopkins $D$, Birkenfeld $A$, et al. Practical recommendations for the management of diabetes in patients with COVID-19. The Lance. Diabetes \& Endocrinology. 2020 Apr 23.

5. OMS. Organización Mundial de la Salud. Informe Mundial Sobre la Diabetes. World Health Organization. 2016.

6. World Health Organization. OMS/ Qué es la diabetes. [Online].; 2020 [cited 202005 08. Available from: https://www.who.int/diabetes/action_online/ basics/es/index1.html.
7. Diaz Rojo JA. El Término Diabetes: aspectos históricos y lexicográficos. Panacea. 2004 Mar; 5(5): p. 15.

8. BBC. Hay 5 tipos de Diabetes y no sólo 2: El estudio que podría cambiar cómo se trata la enfermedad que afecta a 1 de cada 11 personas en el mundo. [Online].; 2018 [cited 202005 08. Available from: http://www.bbc.com/mundo/noticias-43257469/.

9. OMS. Temas de Salud: Diabetes. [Online].; 2016 [cited 202005 08. Available from: https://www. who.int/topics/diabetes_mellitus/es/.

10. Diariofarma. Enfermedad por coronavirus, COVID-19: Información Científico-Técnica. [Online].; 2020 [cited 202005 8. Available from: https://www. diariofarma.com/2020/03/27/enfermedad-por-coronavirus-covid-19-información-cientifica-tecnica/.

11. Bornstein S, Dalan R, Hopkins D, Mingrone G, Boehm B. El vínculo endócrino y metabólico de COVID-19. IntraMed. 2020 Mar.

12. Griner p. Treatment of Acute Pulmonary Edema: Conventional or Intensive Care? Ann Intern Med. 1972; 77: p. 501-506.

13. Camacho Pulido JA, Jurado Lara B, Jiménez Sánchez JM, Montijano Vizcaíno A, Molina Ortega. Síndrome de distrés respiratorio del adulto secundario a inhalación de cocaína. Medicina Intensiva. 2004 Dec; 28(9).

14. Bindom S, Lazartigues $\mathrm{E}$. The sweeter side of ACE2: Physiological evidence for a role in diabetes. Mol Cell Endocrinol. 2009; 302: p. 193-202.

15. Remuzzi A, Remuzzi G. COVID-19 and Italy: What next? The Lancet. 2020; 395: p. 1225-1228.

\section{CITAR ESTE ARTICULO:}

Pinos Robalino, P. J., Segovia Palma, P., Cedeño Delgado, M. J., \& Gonzabay Bravo, E. M. (2020). La diabetes y las complicaciones con el COVID-19. RECIMUNDO, 4(4), 509-516. https://doi.org/10.26820/recimundo/4.(4).octubre.2020.509-516

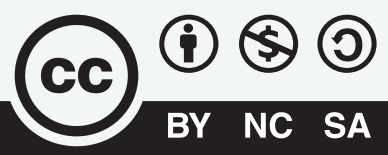

CREATIVE COMMONS RECONOCIMIENTO-NOCOMERCIAL-COMPARTIRIGUAL 4.0. 